(C) [2011] IEEE. Reprinted, with permission, from [Phyo Phyo San, S. H. Ling Member and H. T. Nguyen, Block based neural network for hypoglycemia detection, Engineering in Medicine and Biology Society,EMBC, 2011 Annual International Conference of the IEEE, Aug. 30 2011-Sept. 3 2011]. This material is posted here with permission of the IEEE. Such permission of the IEEE does not in any way imply IEEE endorsement of any of the University of Technology, Sydney's products or services. Internal or personal use of this material is permitted. However, permission to reprint/republish this material for advertising or promotional purposes or for creating new collective works for resale or redistribution must be obtained from the IEEE by writing to pubs-permissions@ieee.org. By choosing to view this document, you agree to all provisions of the copyright laws protecting it 


\title{
Block Based Neural Network for Hypoglycemia Detection
}

\author{
Phyo Phyo San, S. H. Ling Member, IEEE and H. T. Nguyen Senior Member, IEEE
}

\begin{abstract}
In this paper, evolvable block based neural network (BBNN) is presented for detection of hypoglycemia episodes. The structure of BBNN consists of a two-dimensional (2D) array of fundamental blocks with four variable inputoutput nodes and weight connections. Depending on the structure settings, each block can have one of four different internal configurations. To provide early detection of hypoglycemia episodes, the physiological parameters such as heart rate and corrected QT interval of electrocardiogram (ECG) signal are used as the inputs of BBNN. The overall structure and weights of BBNN are optimized by an evolutionary algorithm called hybrid particle swarm optimization with wavelet mutation (HPSOWM). The optimized structures and weights of BBNN are capable to compensate large variations of ECG patterns caused by individual and temporal difference since a fixed structure classifiers are easy to fail to trace ECG signals with large variations. The ECG data of 15 patients are organized into a training set, a testing set and a validation set, each of which has randomly selected 5 patients. The simulation results shows that the proposed algorithm, BBNN with HPSOWM can successfully detect the hypoglycemic episodes in T1DM in term of testing sensitivity (76.74\%) and test specificity (50.91\%).
\end{abstract}

\section{INTRODUCTION}

Current technologies used in the diabetes diagnostic testing and self-monitoring market have already been improved to some extent. However technology advancement in the market is expected to have non-invasive glucose meter with the use of novel design concepts. There is a limited number of non-invasive blood glucose monitoring systems currently available in the market but each of them has its own drawbacks in terms of functioning, cost, reliability and obtrusiveness. Intensive research has been devoted to the development of hypoglycemia alarms, exploiting principles that range from detecting changes in the electroencephalogram (ECG) or skin conductance (due to sweating) to measurements of subcutaneous tissue glucose concentrations by glucose sensors. However, none of these have proved sufficiently.

To carry out modeling and classification for medical diagnose purposes of ECG and EEG [1] much of attention have been devoted to computational technologies such as fuzzy system [2], support vector machine [3] and neural

The authors would like to thank Dr. Nejhdeh Ghevondian, and Assoc. Prof. Timothy Jones for their contribution. This works was supported by a grant from Juvenile Diabetes Research Foundation.

P.P. San is with the Centre for Health Technologies, Faculty of Engineering and IT, University of Technology Sydney, Ultimo, NSW, Australia.e-mail: PhyoPhyo. Sandstudent.uts.edu.au

S.H. Ling is with the Centre for Health Technologies, Faculty of Engineering and IT, University of Technology Sydney, Ultimo, NSW, Australia.e-mail: Steve.Ling@uts.edu.au; phone: +61 (2) 95142390

H.T. Nguyen is with the Centre for Health Technologies, Faculty of Engineering and IT, University of Technology Sydney, Ultimo, NSW, Australia.e-mail: Hung.Nguyen@uts.edu.au networks [4]. Each technology has its own advantages, for example, fuzzy system is famous because of its decision making ability. Due to its human experts representation, the system's output can be determined by a set of linguistic rules which can be understood with an easy manner. In [5], [6] neural network (NNs) has been applied to develop classification models for medical diagnosis due to it's merit of generalization ability in addressing both nonlinear and fuzzy nature of patients data. Support vector machine (SVM) has been used in classification of cardiac signal [7], [8] according to its special feature in tackling binary classification problems. To generate classification models for heart disease [9] and Parkinson's disease [10] in term of polynomial forms, the genetic programming with least square algorithm [11] has been used. According to fuzziness of measures, it is unavoidable that the patients' data involves uncertainty. Since the genetic programming with least square algorithm does not consider the fuzziness of uncertainty during measurement, it cannot give the best classification model for diagnosis purpose.

For modeling and design of a non-invasive hypoglycemia monitor with physiological responses, a significant contribution is devoted in this paper, by using evolvable block based neural networks (BBNN). A hybrid particle swarm optimization based block based neural network (BBNN) is developed for early detection of hypoglycemic episodes by using psychological parameters. In the proposed system, the psychological parameters, HR and QTc are used as the inputs because of its higher correlation with hypoglycemia. Since the classifier with fixed structure [3], [5] are often fail to trace the large variation in ECG signal, BBNN with evolvable structures and weights [14] are proposed due to its merit in approximation of higher correlation between the physiological parameters (HR and QTc). To optimize the structure and weights of BBNN, a powerful random global learning optimization algorithm called hybrid particle swarm optimization with wavelet mutation (HPSOWM) [13] is introduced. The structure and weights of BBNN are optimized by the use of training data and the optimized BBNN is

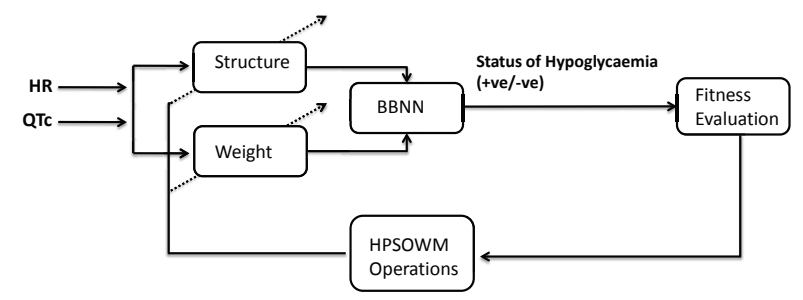

Fig. 1. PSO based block based neural network for hypoglycaemia detection 
expected to give higher performance in terms of sensitivity and specificity. The organization of this chapter is as follows: In Section II, block based neural networks and their training procedures by the use of hybrid particle swarm optimization with wavelet mutation (HPSOWM) is introduced. To show the effectiveness of our proposed methods, the results of early detection of nocturnal hypoglycemic episodes in T1DM are discussed in Section III before a conclusion is drawn in Section IV.

\section{METHODS}

To realize the detection of hypoglycemic episodes in T1DM, an evolvable block based neural network (BBNN) with 2 inputs and 1 output system is developed for the early detection of hypoglycemia episodes as shown in Figure. 1. The two inputs are the psychological input: the heart rate (HR) and the corrected QT interval (QT) of electrocardiogram (ECG) signal while the output is the presence of hypoglycemia (h) in which +1 represents hypoglycemia and -1 is non-hypoglycemia. In this proposed system, the structure and the weights of BBNN are optimized by HPSOWM [13] and the detail structure of BBNN with HPSOWM are presented in this section.

\section{A. Block Based Neural Network (BBNN)}

A block based neural network (BBNN) [14], [16] is a network design that is more flexible to change the structure depending on the signal flow between blocks. In [15], it has been proposed an effective network structure to solve ECG classification problem due to the significant improvement of the sensitivity, specificity and accuracy rate. It can be represent by 2-D array of blocks and each individual neuron blocks work as a basic signal processing unit that is composed of a feedforward neural network having four variable input/output nodes. A block is connected to its neighboring blocks with signal flow represented by arrows: $\downarrow$ represent as 0 while $\uparrow$ and $\rightarrow$ assign as 1 . As shown in Fig. 2, the structure of BBNN is organized with $m$ rows and $n$ columns of blocks which is labeled as $B_{i j}$, in which $(i=1, \ldots m)$ and $(j=1, \ldots, n)$.

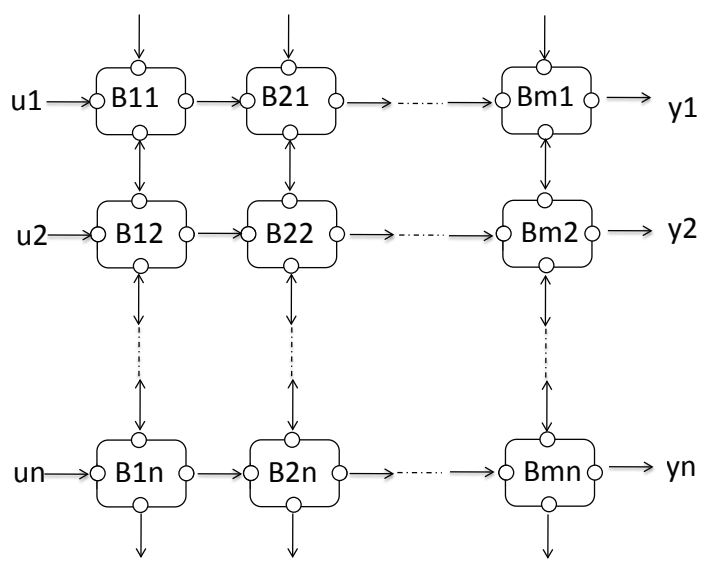

Fig. 2. Structure of Block-Based Neural Network
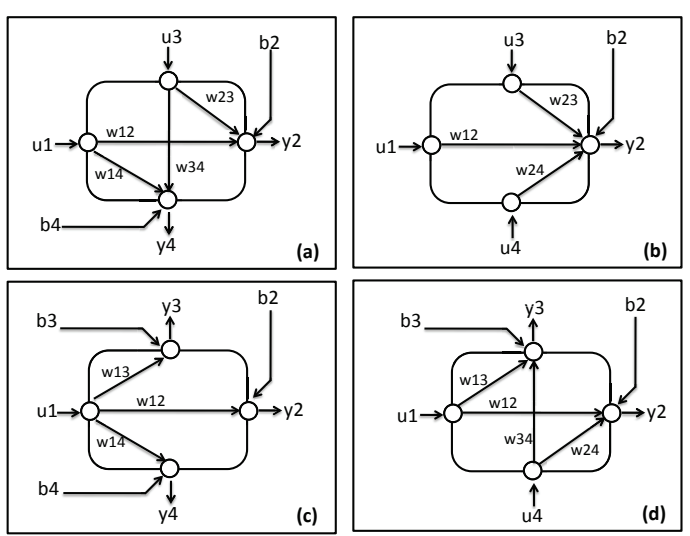

Fig. 3. Internal configurations of block based neural network

The first column of blocks, $B_{11}, B_{12}, \ldots, B_{1 m}$ is determined as the input layer of BBNN network structure while the last column of blocks, $B_{m 1}, B_{m 2}, \ldots, B_{m n}$ is represented as output layer. The output of BBNN network $\left(y=y_{1}, y_{2}, y_{3}, \ldots, y_{n}\right)$ is a function of summation of weighted inputs and a bias. A constant input value is given to redundant input nodes and un-used output nodes are ignored. Due to modular characteristics, BBNN can be easily expanded to be a larger network. In this paper, the connections between layers will only be considered as feedforward configuration in forward direction. The structure of BBNN is determined by automatically internal configuration or input-output connection of basic blocks. According to the input-output connections of the network structure, the block has four different types of internal configuration. Fig. 3 (a) and (d) represent for two input and two output with different internal configurations while (b) and (c) correspond to one input-three outputs and three-output configurations. The capability of generalization is improved through various internal configurations of a block. Even though each basic block should be one input and three output (1/3), three inputs and one output (3/1), two inputs and two outputs $(2 / 2)$, the extreme cases of all input nodes (4/0) or all output nodes (0/4) are considered as invalid configurations.

For each basic block consists of four nodes and each nodes inside the block are connected with each other through connection of weights, $w_{i j}$. A block can have up to maximum of six connection weights including the biases. For the case of two inputs and two outputs in Fig. 3 (b) and (d), there are four weights and two biases. Same as the case in Fig. 3 (c), one input and three outputs structure has three weights and and three biases. For three inputs and one output cased in Fig. 3 (a), it belongs to three weights and one biases. Each node of a block can be an input or an output according to internal configuration which is determined by the signal flow. An incoming arrow to a block is defined as the input nodes and its associated outputs are considered as the block outputs with outgoing arrows. The output of each block is connected to the another block as the input signal. The output of the block is calculated by the summation of weighted inputs and 
biases corresponded to a feedforward NN as follows:

$$
y_{j}=\sum_{i \in I} w_{i j} u_{i}+b_{j}, \quad j \in J
$$

where $x_{i}$ is the input to node $i, b_{J}$ is the biases of $j, I$ and $J$ are the two index set for input and output nodes. For $(1 / 3)$ block $I=1$ and $J=2,3,4$. For block type of $(3 / 1), I=1,3,4$ and $J=2$ while the $(2 / 2)$ block type has $I=1,4$ and $J=2,3$. For each node, it is characterized by the following activation function, $\varphi(\cdot)$ :

$$
\varphi(u)=a\left(\frac{2}{1+e^{-b u}}-1\right)
$$

where the parameter $a$ and $b$ will be optimized by HPSOWM in [13].

\section{B. Hybrid Particle Swarm Optimization with Wavelet Muta- tion (HPSOWM)}

In HPSOWM, a swarm $X(t)$ is constituted with the number of particles. Each particle $\mathbf{x}^{p}(t) \in X(t)$ contains $\kappa$ elements $x_{j}^{p}(t)$ at the $t$-th iteration, where $p=1,2, \ldots, \theta$ and $j=1,2, \ldots, \kappa ; \theta$ denotes the number of particles in the swarm and $\kappa$ is the dimension of a particle. First, the particles of the swarm are initialized and then evaluated by a defined fitness function. The objective of HPSOWM is to minimize the fitness function (cost function) $f(X(t))$ of particles iteratively. The position $x_{j}^{p}(t)$ and velocity $v_{j}^{p}(t)$ used in HPSOWM [13] are given as follows:

$$
\begin{aligned}
x_{j}^{p}(t) & =x_{j}^{p}(t-1)+v_{j}^{p}(t) \\
v_{j}^{p}(t) & =k \cdot\left(w \cdot v_{j}^{p}(t-1)+\varphi_{1} r_{1}\right) \cdot\left(\tilde{x}_{j}-x_{j}^{p}(t-1)\right) \\
& +\varphi_{2} r_{2}\left(\hat{x}_{j}-x_{j}^{p}(t-1)\right)
\end{aligned}
$$

where $\tilde{x}^{p}=\left[\tilde{x}_{1}^{p}, \tilde{x}_{2}^{p}, \ldots, \tilde{x}_{k}^{p}\right]$ and $\hat{\mathbf{x}}=\left[\begin{array}{lll}\hat{x_{1}} & \hat{x_{2}} & , \ldots \hat{x_{\kappa}}\end{array}\right], j=$ $1,2, \ldots, \kappa$. The best previous position of a particle is recorded and represented as $\tilde{x}$; the position of best particle among all the particles is represented as $\hat{x} ; w$ is an inertia weight factor; $r_{1}$ and $r_{2}$ are acceleration constants which return a uniform random number in the range of $[0,1] ; w$ is inertia weight factor and $k$ is a constriction factor which detail derivation is discussed in [13].

$$
\bar{x}_{j}^{p}(t)=\left\{\begin{array}{cc}
x_{j}^{p}(t)+\sigma \times\left(\rho_{\max }^{j}-x_{j}^{p}(t)\right) & , \sigma>0 \\
x_{j}^{p}(t)+\sigma \times\left(x_{j}^{p}(t)-\rho_{\min }^{j}\right) & , \sigma<0
\end{array}\right.
$$

where $j \in 1,2, \ldots, \kappa$ and $\kappa$ denotes the dimension of particles. The value of $\sigma$ is governed by Morlet wavelet function as presented in [13].

\section{Fitness Function and Training}

The performances of classifications are measured in terms of sensitivity and specificity. The performance of proposed detection system is measured in terms of Sensitivity, $\xi=$ $\frac{N_{T P}}{N_{T P}+N_{F N}}$ and Specificity, $\eta=\frac{N_{T N}}{N_{T N}+N_{F P}}$ in which $N_{T P}$ is defined as number of true positive which implies the sick people correctly diagnosed as sick; $N_{F N}$ is number of false negative which implies the sick people wrongly diagnosed as healthy; $N_{F P}$ is number of false positive which implied healthy people wrongly diagnosed as sick; and $N_{T N}$ is number of true negative which implied healthy people correctly diagnosed as healthy. The values of these are within 0 to 1 . The objective is to maximize the fitness function of (5) which equivalent to maximize the sensitivity and the specificity. To meet the objective of the system the fitness function $f(\xi, \eta)$ is defined as follow:

$$
f(\xi, \eta)=\xi+\frac{\eta_{\max }-\eta}{\eta_{\max }}
$$

where $\eta_{\max }$ is a upper limit of the specificity. In this papers, each particle of HPSOWM contains the structure $\left(s_{l}\right)$ and weights $\left(w_{k}^{l}\right)$ parameters of BBNN and transfer function parameters $a$ and $b$ from eq. (2) which is in the form of $\mathbf{x}=\left[\begin{array}{llll}s_{l} & w_{k}^{l} & a & b\end{array}\right]$, where $l$ and $k$ are number of layers and weights of BBNN (up to 12 connection weights for each layer). In (5), the specificity is limited by a maximum value $\eta_{\max }$. The parameter $\eta_{\max }$ is used to fix the region of specificity and find the optimal sensitivity in this region. In particularly, the $\eta_{\max }$ can set from 0 to 1 and different sensitivity with different specificity value can be determined.

\section{RESULT AND DISCUSSION}

To study the natural occurrence of nocturnal hypoglycemia, 15 children with T1DM is monitored for the 10hour overnight at the Princess Margaret Hospital for Children in Perth, Western Australia, Australia. The required physiological parameters are measured by the use of non-invasive monitoring system in [20], while the actual blood glucose levels (BGL) are collected as reference using Yellow Spring Instruments. The main parameters which is used for the detection of hypoglycemia are the heart rate (HR) and corrected QT (QTc) interval. The responses from 15 children with T1DM exhibit significant changes during the hypoglycemia phase against the non-hypoglycemia phase. Normalization is used to reduce patient-to-patient variability and to enable group comparison by dividing the patient's heart rate and corrected QT interval by his/her corresponding values at time zero. The study shows that the hypoglycemia episodes (BGL $\leq 3.3 \mathrm{mmol} / \mathrm{l}$ ) is detected using these variables based on a hybrid PSO with wavelet mutation based block based neural network model. The overall data set consisted of both hypoglycemia data part and non-hypoglycemia data part and it is organized into a training set (5 patients), a validation set (5 patients) and a testing set (5 patients) which are randomly selected.

To find the optimized structure and weights of BBNN, the parameters of HPSOWM are chosen as: swarm size $\theta=50$, constant value $c_{1}$ and $c_{2}=2.05$, maximum velocity $v_{\max }=$ 0.3 , probability of mutation $\mu_{m}=0.7$, the shape parameter of wavelet mutation $\zeta_{w m}=2$, the constant value $g$ of wavelet mutation $=10000$ and the number of iteration $T=2000$.

In Table I, the results are evaluated in terms of mean values in which all the results are calculated by averaging over 20 sets of data. Since the sensitivity mainly represents patients with hypoglycaemia, in order to analyse the performance 
TABLE I

Mean Value of Training, Validation and Testing Results: SPECIFICITY $($ TRAINING $)=40 \%$

\begin{tabular}{|l|cc|cc|ccc|}
\hline Method & \multicolumn{2}{|c|}{ Training } & \multicolumn{2}{|c|}{ Validation } & \multicolumn{3}{c|}{ Testing } \\
& Sen & Spec & Sen & Spec & Sen & Spec & gm \\
\hline \hline BBNN & $83.64 \%$ & $40.35 \%$ & $87.34 \%$ & $40.13 \%$ & $76.28 \%$ & $52.40 \%$ & $63.22 \%$ \\
\hline FWNN & $81.06 \%$ & $41.26 \%$ & $76.41 \%$ & $41.44 \%$ & $65.93 \%$ & $50.09 \%$ & $57.46 \%$ \\
\hline MR & $85.76 \%$ & $40.89 \%$ & $73.28 \%$ & $40.00 \%$ & $63.37 \%$ & $59.74 \%$ & $61.52 \%$ \\
\hline
\end{tabular}

TABLE II

Best Testing Result for Hypoglycemia Detection With DIFFERENT APPROACHES

\begin{tabular}{|c|c|c|c|}
\hline Method & Sensitivity(Testing) & Specifcity(Testing) & gm \\
\hline \hline BBNN & $76.74 \%$ & $50.91 \%$ & $63.06 \%$ \\
\hline FWNN & $67.44 \%$ & $54.55 \%$ & $60.65 \%$ \\
\hline MR & $62.79 \%$ & $60.91 \%$ & $61.84 \%$ \\
\hline
\end{tabular}

of sensitivity, the specificity of training process are set to $40 \%$. By doing so, the average (mean) testing result of proposed BBNN model gives satisfactory sensitivity, $76.28 \%$ and specificity, $52.40 \%$ which is outperformed than the other models such as FWNN and MR whose sensitivity and specificity are $(65.93 \%$ and $50.09 \%)$ and $(63.67 \%$ and $59.74 \%)$ respectively. In addition, the performance of proposed BBNN are assessed in terms of geometric mean, $(g m=\sqrt{\xi \times \eta})$. In Table I, the proposed BBNN approach performs approximately $2 \%$ and $3 \%$ better than that of FWNN and MR approaches.

After the training process, the optimized block based neural network (BBNN) model gives the best testing sensitivity, $76.74 \%$ and acceptable specificity, $50.91 \%$ as tabulated in Table II. The obtained BBNN result is significantly improved compared with conventional classification techniques such as feedforward neural network (FWNN) $(67.44 \%$ of testing sensitivity and $54.55 \%$ of testing specificity) and multiple regression (MR) $(62.79 \%$ of testing sensitivity and $60.91 \%$ of testing specificity). Besides, the proposed BBNN gives better geometric mean value of $63.06 \%$ while FWNN and MR only have $60.65 \%$ and $61.84 \%$.

\section{CONCLUSIONS}

A hybrid particle swarm optimization based block based neural network (BBNN) is developed for detect the hypoglycemia episodes for diabetes patients. The result in Section III indicate that the hypoglycemia episodes in T1DM children can be detected non-invasively and continuously from the real-time physiological responses (heart rate,corrected QT interval). To optimize the structure and weight of BBNN, a hybrid particle swarm optimization is presented where wavelet mutation operation is introduced to enhance the optimization performance. An actual T1DM study illustrated that the proposed algorithm gives better sensitivity and specificity than other conventional algorithms, feedforward and multi regression. In short, the proposed PSO based BBNN detection system is effective by giving $76.74 \%$ of satisfactory sensitivity and $50.91 \%$ of specificity.

\section{REFERENCES}

[1] C. M. Fira and L. Goras, "An ECG signals compression method and its validation using NNs", IEEE Transactions on Biomedical Engineering, vol.55, no. 4, pp 1319-1326, 2008.

[2] J. S. Jang and C. T. Sun, Neuro-Fuzzy and Soft Computing: A Computational Approach to Learning and Machine Intelligence, Prentice Hall, 1997.

[3] C. Cortes and V. Vapnik, "Support-vector networks", Machine Learning, vol.20, no. 3, pp 273-297, 1995.

[4] A. K. Jain and J. Mao and K. M. Mohiuddin, "Artificial neural networks: a tutorial", IEEE Computer Society, vol.29, no. 3, pp 3144, 1996.

[5] M. Brown and C. Harris, Neural Fuzzy Adaptive Modeling and Control, Prentice Hall, 1994.

[6] J.A. Reggia and S.S. Sutton, "Self-processing networks and their biomedical implications", Proceedings of the IEEE, vol.76, no. 6, pp $580592,1988$.

[7] D. Meyer, F. Leisch, and K. Hornik, "The support vector machine under test", Neurocomputing, vol.55, no. 6, pp 169186, 2003.

[8] N. Acir, "A support vector machine classifier algorithm based on a perturbation method and its application to ECG beat recognition systems”, Expert Systems with Applications, vol. 31, no. 1, pp 150158, 2006.

[9] S. M. Winkler, M. Affenzeller, and S. Wagner, "Using enhanced genetic programming techniques for evolving classifiers in the context of medical diagnosis", Genetic Programming and Evolvable Machines, vol. 10, no. 2, pp 111-140, 2009.

[10] T. S. Subashini, V. Ramalingam, and S. Palanivel, "Breast mass classification based on cytological patterns using RBFNN and SVM", Source Expert Systems with Applications: An International Journal archive, vol. 36, no. 3, 2009, pp 5284-5290.

[11] H. F. Gray and R. J. Maxwell, "Genetic programming for classification and feature selection: analysis of $1 \mathrm{H}$ nuclear magnetic resonance spectra from human brain tumour biopsies", NMR in Biomedicine, vol. 11, no. 4, pp 217224, 1998.

[12] M. L. Astion, M. H. Wener, and R. G Thomas and G. G Hunder, "Overtraining in neural networks that interpret clinical data", Clinical Chemistry, vol.39, no.9, pp 1998-2004, 1993.

[13] S. H. Ling, H. C. C. Iu, K. Y. Chan, H. K. Lam, C. W. Yeung, and F. H. F Leung, "Hybrid particle swarm optimization with wavelet mutation and its industrial applications", IEEE transactions on systems, man, and cybernetics. Part B, vol.38, no.3, pp 743763, 2008.

[14] S. W. Moon and S. G. Kong, "Block-based neural networks", IEEE Trans Neural Network, vol. 12, pp 307-317, 2001.

[15] W. Jiang and G. Seong Kong, "Block-Based Neural Networks for Personalized ECG Signal Classification", IEEE Transactions on Neural Networks, vol. 18, no. 6, pp 1750-1761, 2004.

[16] S. W. Moon and S. G. Kong, "Pattern recognition with block-based neural networks", International Joint Conference on Neural Networks, pp 992 - 996, 2002.

[17] R. C. Eberhart and Y. Shi, "Comparing inertia weights and constriction factors in particle swarm optimization", Proceedings of IEEE Congress on Evolutionary Computing, pp 84-88, 2000.

[18] Z. Michalewicz, Genetic algorithms + data structures = evolution programs (2nd, extended ed.), Springer-Verlag, 1994.

[19] D. G. Altman and J. M. Bland, "Statistics Notes: Diagnostic tests 1: sensitivity and specificity", Clinical chemistry, vol.308, pp 15521552, 1994.

[20] H. T. Nguyen, N. Ghevondian, and T. W. Jones, "Neural-Network Detection of Hypoglycemic Episodes in Children with Type 1 Diabetes using Physiological Parameters", Proceedings of 28th Annual International Conference of the IEEE Engineering in Medicine and Biology Society, pp 6053-6056, 2006. 